\title{
Terrestrial Planet Finder Interferometer: 2007-2008 progress and plans
}

P. R. Lawson, O. P. Lay, S. R. Martin, R. D. Peters, R. O. Gappinger, et al.

P. R. Lawson, O. P. Lay, S. R. Martin, R. D. Peters, R. O. Gappinger, A. Ksendzov, D. P. Scharf, A. J. Booth, C. A. Beichman, E. Serabyn, K. J. Johnston, W. C. Danchi, "Terrestrial Planet Finder Interferometer: 2007-2008 progress and plans," Proc. SPIE 7013, Optical and Infrared Interferometry, 70132N (28 July 2008); doi: 10.1117/12.786822

EDIE Event: SPIE Astronomical Telescopes + Instrumentation, 2008, Marseille, France 


\title{
Terrestrial Planet Finder Interferometer 2007-2008 Progress and Plans
}

\author{
P. R. Lawson ${ }^{1}$, O. P. Lay ${ }^{1}$, S. R. Martin ${ }^{1}$, R. D. Peters ${ }^{1}$, R. O. Gappinger ${ }^{1}$, A. Ksendzov ${ }^{1}$, \\ D. P. Scharf ${ }^{1}$, A. J. Booth ${ }^{1}$, C. A. Beichman ${ }^{2}$, E. Serabyn ${ }^{1}$, \\ K. J. Johnston ${ }^{3}$, W. C. Danchi4 \\ ${ }^{1}$ Jet Propulsion Laboratory, California Institute of Technology, Pasadena, CA, USA \\ ${ }^{2}$ Michelson Science Center, California Institute of Technology, Pasadena, CA, USA \\ ${ }^{3}$ US Naval Observatory, Washington, DC, USA \\ 4NASA Goddard Space Flight Center, Greenbelt, MA, USA
}

\begin{abstract}
This paper provides an overview of technology development for the Terrestrial Planet Finder Interferometer (TPF-I). TPF-I is a mid-infrared space interferometer being designed with the capability of detecting Earth-like planets in the habitable zones around nearby stars. The overall technology roadmap is presented and progress with each of the testbeds is summarized.
\end{abstract}

Keywords: Interferometry, astronomy, extrasolar planets, nulling, formation flying

\section{INTRODUCTION AND OVERVIEW}

The Terrestrial Planet Finder Interferometer (TPF-I) is a concept for a formation-flying interferometer designed to measure mid-infrared spectra of the atmospheres of Earth-like exoplanets. The primary goal of the mission is to find evidence of biological activity on planets around nearby stars. The mid-infrared provides several key biomarkers and a favorable planet-star contrast ratio. An interferometer is a compelling choice for the design of a such a mid-infrared observatory because the interferometric baselines provide unrivaled angular resolution. This is vital for unambiguous orbit determination, distinguishing multiple planets, and discrimination against structure in the exozodiacal disk. This angular resolution also provides a small inner working angle, giving access to a very broad range of target stars. In this regard, TPF-I far exceeds the predicted capability of other proposed planet-finding missions. TPF-I has been developed as a possible future collaboration between NASA and the European Space Agency (ESA).

This paper provides an update to the reviews presented at SPIE conferences in $2006^{1}$ and $2007 .{ }^{2}$ The major achievements and/or changes in 2007-2008 have included the following:

- Completion of Milestone \#1: the compensation of intensity and phase demonstrated by the Adaptive Nuller testbed. Intensity was compensated to within $0.2 \%$ and phase to within $5 \mathrm{~nm}$ rms across a $3-\mu \mathrm{m}$ band centered at $10-\mu \mathrm{m} .{ }^{3,4}$

- Completion of Milestone \#2: a demonstration of precision formation maneuvers in a ground-based robotic testbed, with performance traceability to flight. ${ }^{5,6}$

- The demonstration of nulling over a $32 \%$ bandwidth centered at a wavelength of $10 \mu \mathrm{m}$ at a level of $1.1 \times 10^{-5}$ using the Adaptive Nuller. ${ }^{4}$

- An increased emphasis on the design of a reduced-scope mission. ${ }^{7}$

- The adoption of the Emma X-Array by both the TPF-I Project and the Darwin proposal team as the baseline mission design for TPF-I and Darwin. ${ }^{8}$

Further author information: Send correspondence to Peter Lawson, Jet Propulsion Laboratory, MS 301-451, 4800 Oak Grove Drive, Pasadena CA 91109-8099, USA. E-mail: Peter.R.Lawson@jpl.nasa.gov, Telephone: +1 (818) 354-0747.

Optical and Infrared Interferometry, edited by Markus Schöller, William C. Danchi, Françoise Delplancke Proc. of SPIE Vol. 7013, 70132N, (2008) · 0277-786X/08/\$18 - doi: 10.1117/12.786822 


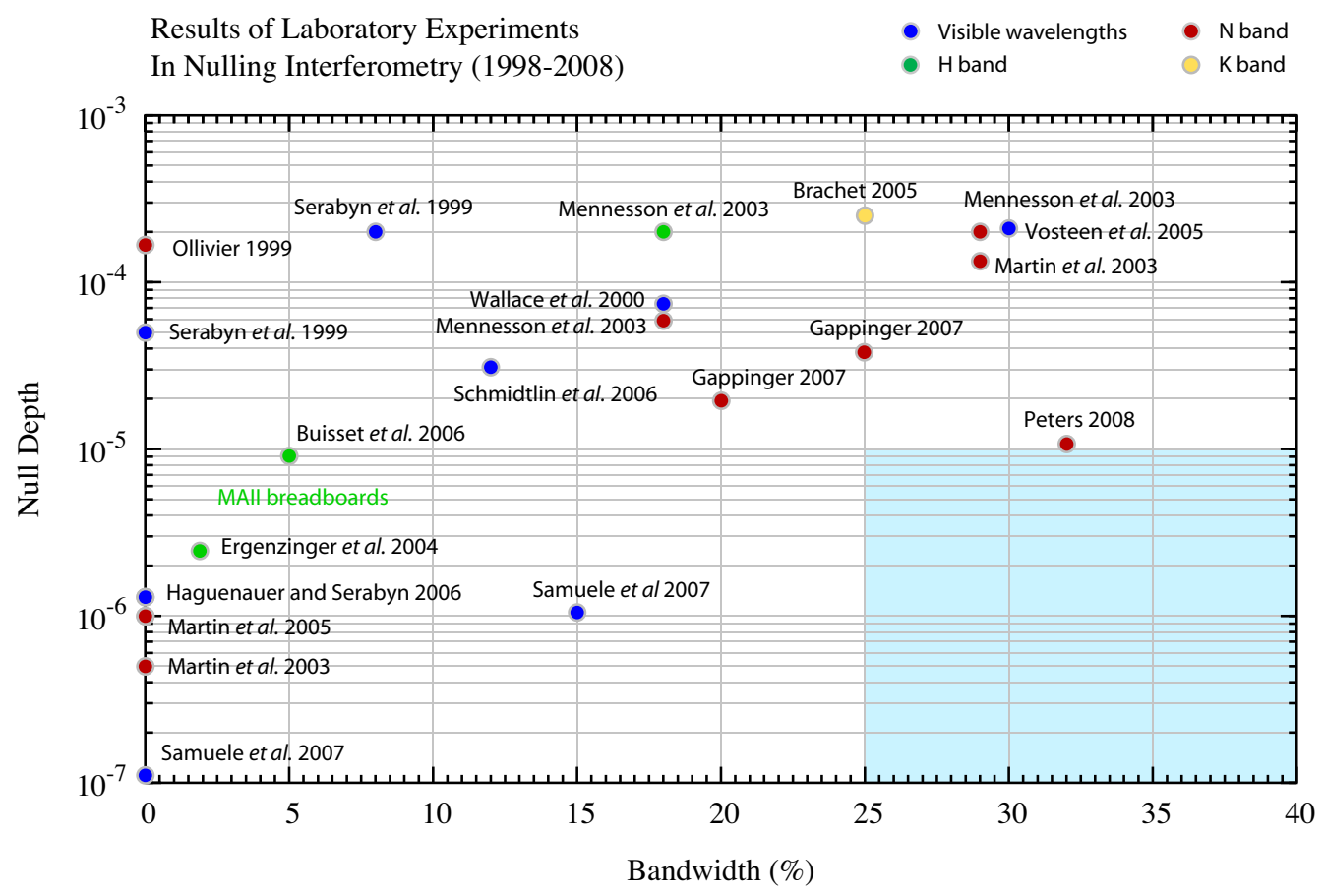

Figure 1. State of the Art in Nulling Interferometry: Laser experiments have shown that achromatic effects (predominantly pathlength variations) can be controlled in the lab at a level that allows nulls better than $1 \times 10^{-6}$ to be achieved repeatedly. The best broadband mid-infrared nulls achieved to date have been $1.1 \times 10^{-5}$, with the Adaptive Nuller. The laser results therefore exceed the TPF-I requirements, and the broadband results are just shy of the TPF-I goal of $1.0 \times 10^{-5}$.

All mission-specific studies ceased in 2007, and all of the testbed activities have been reduced in scope. The results of most testbed work accomplished to date has now been submitted for publication in refereed journals. In 2008 the focus of continuing work is on broadband adaptive nulling (Milestone \#3) and a system-level demonstration of planet detection by four-beam array rotation with chopping and averaging (Milestone \#4). As of September 2008, TPF will cease to exist as a NASA project in Pre-formulation Phase, although a subset of its activities will continue as pure technology development.

\section{TECHNOLOGY FOR STARLIGHT SUPPRESSION}

Progress in nulling interferometry is summarized in Fig. 1. The plot shows null depth as a function of bandwidth, for laboratory experiments that have been reported since $1999 .^{9-28}$ On the far left-hand side of the plot are shown the results obtained using lasers at visible, near-infrared, and mid-infrared wavelengths. Experiments with bandwidths as large as $40 \%$ are shown. Of principal concern to TPF-I are the experiments that have been conducted at mid-infrared wavelengths, which are indicated by the red circles in the plot. The light blue region is the performance required of TPF-I testbeds, being rejection ratios (inverse of the null depth) better than 100,000:1 with a bandwidth of $25 \%$ or larger. The reported results are almost all limited to a single-polarization input. All but one of the results are from two-beam nulling interferometers. From the results to date, we can draw the following conclusions.

1. Laser experiments have shown that achromatic effects (predominantly pathlength variations) can be controlled in the lab at a level that allows rejection ratios better than 1,000,000:1 to be achieved repeatedly. This level of performance exceeds the requirements for TPF-I. Narrow-bandwidth laser nulls have been attained with mid-infrared rejection ratios of 2,000,000:1 and at visible wavelengths of 10,000,000:1. 
2. The best broad-band mid-infrared results were obtained in June 2008 with the Adaptive Nuller tesbed. ${ }^{4}$ A rejection ratio of 93,000:1 was obtained with a $32 \%$ bandwidth. This rejection ratio is very close to $100,000: 1$ and therefore almost at the level of the flight requirements. The approach used by the Adaptive Nuller is noteworthy because it is not intrinsically bandwidth limited; a spectrum is imaged onto a line of pixels on a deformable mirror to correct phase and intensity across the band, and this method can easily be extended to work well across the full 6-20 $\mu \mathrm{m}$ science band.

If one takes into account the relaxation of requirements due to advances in our understanding of instability noise ${ }^{29}$ the best broad-band mid-infrared null have nearly demonstrated the flight requirements for TPF-I. Work is ongoing to achieve deeper nulls and to demonstrate similar capability with a four-beam testbed. ${ }^{30}$

\subsection{Mid-IR Spatial Filter Development}

Spatial filters are an essential technology for nulling interferometry. They significantly reduce the optical aberrations in wavefronts, making extremely deep nulls possible. The most basic form of a spatial filter is a simple pinhole, and pinholes have indeed been used to achieve the deepest laser nulls so far at mid-infrared wavelengths. However, pinholes only operate well over a narrow bandwidth and so are ill-suited for broadband spatial filtering for science instruments. In addition, they do not reject very low spatial-frequency wavefront aberrations. The development of improved broadband techniques for spatial filtering at mid-infrared wavelengths may be crucial to the success of TPF-I, and has been a focus of research at JPL. Although fiber optics at near-infrared wavelengths are extensively used by the telecommunications industry, low-loss, mid-IR, single-mode spatial filters are not yet commercially available. The goal of this work has been to develop single-mode spatial filters with a throughput of $50 \%$ or better with a modal suppression of $25 \mathrm{~dB}$ for non-fundamental modes.

\subsubsection{Description of Research}

Spatial filters may be implemented in a variety of ways, including single-mode fiber-optics made from chalcogenide or silver halide glasses, metallized waveguide structures micro-machined in silicon, or through the use of photonic crystal fibers. By promoting the parallel development of various spatial filter technologies, it was hoped that we would demonstrate the necessary spatial filter performance in the 6-20 $\mu \mathrm{m}$ spectrum using no more than two technology types. The development of mid-infrared spatial filters was funded by TPF-I from 2003 through 2008. The performance of the single-mode filters that were developed under contract with TPF-I were tested and characterized in-house at JPL. The scope of the work included prototypes of hollow waveguide filters designed by Christopher Walker at the University of Arizona; completed chalcogenide fibers designed by Dr. Jas Sanghera at the Naval Research Laboratory (NRL); and completed silver halide fibers designed by Prof. Abraham Katzir at Tel Aviv University (TAU) in Israel.

\subsubsection{Results}

The results of this research have been published by Ksendzov et al. ${ }^{31,32}$ and Tomer et al. ${ }^{33}$ The fibers that have been developed for TPF-I represent the state of the art. About 16 mid-infrared single-mode fibers were delivered and tested at JPL, showing excellent single-mode behavior at $10 \mu \mathrm{m}$.

The 20-cm-long chalcogenide fibers developed by the Naval Research Laboratory were shown to demonstrate $30 \mathrm{~dB}$ rejection (a factor of 1000) of higher order modes and have an efficiency of $40 \%$, accounting for both throughput and Fresnel losses. The transmission losses were measured at $8 \mathrm{~dB} / \mathrm{m}$, and the fibers are usable up to a wavelength of about $11 \mu \mathrm{m}$. The chalcogenide fibers developed at the Naval Research Laboratory were used in the Achromatic Nulling Testbed and continue to be used in the Adaptive Nuller testbed (see Fig. 5).

The 10-20 cm long silver halide fibers that were developed by Tel Aviv University were shown to demonstrate $42 \mathrm{~dB}$ rejection (a factor of 16,000) of higher order modes with transmission losses of $12 \mathrm{~dB} / \mathrm{m}$. This high rejection of higher-order modes was accomplished with the addition of aperturing of the output of the fibers, made possible by the physically large diameter of the fiber cladding. Silver halide fibers should in principle be usable up to a wavelength of about $18 \mu \mathrm{m}$, although the laboratory tests at JPL were conducted only at $10 \mu \mathrm{m}$. This is the first time silver halide fibers were shown to have single-mode behavior. 


\subsubsection{Timeline}

Work on hollow waveguides, begun in 2003, ceased in 2005, when it became apparent that single-mode fibers would be the most viable technology to support. The contract work with the Naval Research Laboratory on Chalcogenide fibers was successfully completed in 2005. Work up until 2007 then focused on maturing the technology for Silver Halide fibers, which was likewise successful, and work was then suspended.

\subsubsection{Future Technology Development}

An important step in future work will be to demonstrate single-mode performance over the entire wavelength band that TPF-I will use $(6-20 \mu \mathrm{m})$. To date, experiments that test single-mode behavior have been limited to a narrow wavelength range near $10 \mu \mathrm{m}$. It would be extremely useful to verify and validate the short-wavelength performance of the chalcogenide fibers and the long-wavelength performance of silver-halide fibers. Although the fibers that were developed worked very well, their throughput was not as good as one might desire for a flight mission. The spatial filtering capabilities of photonic crystal fibers should be investigated for use at midinfrared wavelengths, because of the improved throughput that they may provide; higher fiber throughput would effectively allow smaller collector spacecraft to be used. Also, at some future date, the suitability of these fibers for use in a cryogenic environment should be tested.

\subsection{Achromatic Phase Shifter Studies}

The Achromatic Nulling Testbed (ANT) was developed to study achromatic phase shifting techniques to achieve, broadband, dual-polarization, two-beam mid-infrared nulls. The two-beam nuller is the basic building block of all flight architectures that have been considered. Three approaches to achromatic phase shifting were investigated, with the aim of demonstrating, through one of the approaches, two-beam nulling to a level of 1 part in 100,000 with a $25 \%$ bandwidth in the 6-20 $\mu \mathrm{m}$ range. A longer-term objective was the development of a cryogenic nulling interferometer that would meet the above requirements while operating at a temperature of $40 \mathrm{~K}$.

\subsubsection{Testbed Description}

Three different methods of implementing achromatic phase shifts were investigated: (1) pairs of dispersive glass plates to introduce a wavelength-dependent phase delay; (2) A through-focus field-flip of the light in one arm of the interferometer; and (3) successive and opposing field-reversals on reflection off flat mirrors in a periscope arrangement. A close-up view of the periscope mirrors is shown in Fig. 2. These methods were tested in the same lab, on adjacent optical benches, using a common mid-infrared laser and white-light source. The overall strategy was to develop an error budget for each approach, model the optics, isolate and measure the contributing noise sources, and compare the achievable null depths with the model predictions.

The optical breadboards were vibrationally isolated from the optical table that they were mounted on, and the table itself was further isolated from the floor. Pathlength fluctuations were monitored and maintained at a level of less than a few nanometers. The Achromatic Nulling Testbed used laser metrology and automated alignment algorithms to scan across the zero path-difference position and locate the interference null. Pathlength dither algorithms were used to locate the null and maintain the stability of the fringes.

In each of the approaches that were considered, an achromatic phase shifts of 180 degrees should be straightforward to accomplish, however in the field-reversal approaches, (2) and (3) above, a pupil-dependent polarization and/or amplitude mismatch can occur, which must then be corrected using spatial filters. Mid-infrared singlemode fibers, described previously, were therefore used with these approaches.

Ancillary optical components and detectors were developed for the Achromatic Nulling Testbed. A new high-flux continuum argon arc lamp was built to increase the dynamic range of the measurements. A view of the recombining optics, showing the intensity-balancing cross-hairs, is given in Fig. 3. Work was also undertaken to improve the low-light level limit of measurements through the development of a 10- $\mu \mathrm{m}$ camera with high dynamic range. Components for the balancing of intensities and phases were also tested. Moreover, as noted previously, new single-mode fibers made from chalcogenide glass were used. 


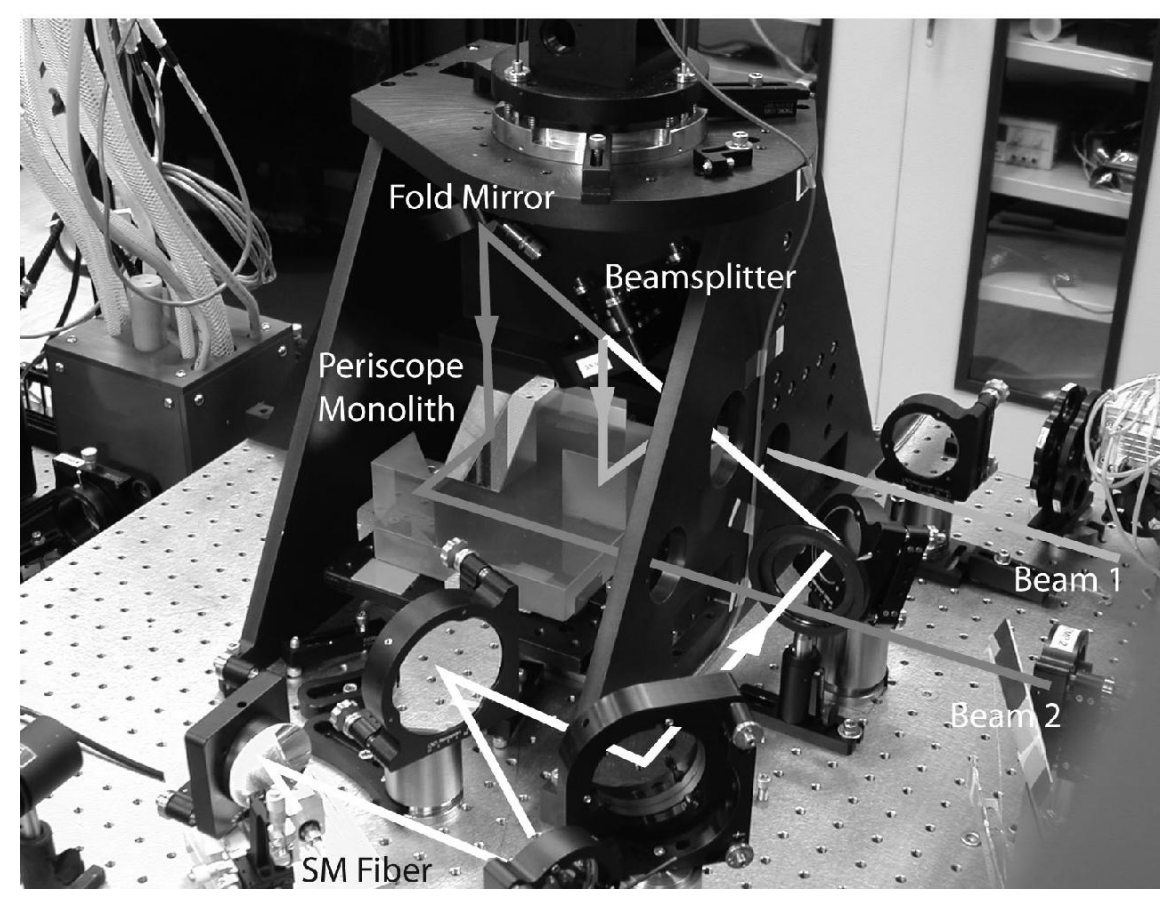

Figure 2. The Achromatic Nulling Testbed (ANT). The ANT included three testbeds to explore technology for achromatic phase shifters in the mid-infrared. The goal of the testbeds was to achieve mid-infrared null depths of 1000,000:1 using a bandwidth of $25 \%$, centered at a wavelength near $10 \mu \mathrm{m}$. The view of the ANT in the above photo shows the periscope assembly. With this approach, mid-infrared nulls of 51,000:1 were achieved in polarized light with a $20 \%$ bandwidth.

\subsubsection{Results}

The results of this work have been summarized by Gappinger et al. ${ }^{34}$ The most successful approach was the use of periscope mirrors, yielding an average rejection ratio of 51,000:1 at 20\% bandwidth and 27,000:1 using a $25 \%$ bandwidth. The through-focus approach yielded a rejection ratio of 2000:1 with a $17 \%$ bandwidth. Pairs of glass plates provided a rejection ratio of $10,000: 1$ with a $25 \%$ bandwidth.

Tests using the same optics, but with narrow-band 10- $\mu$ m laser light and mid-infrared polarizers routinely yielded null depths of 200,000:1. The laser nulling results suggested that a factor of 4 improvement in broadband nulling was possible. There were undoubtedly wavelength-dependent effects that were different in one arm of the interferometer than in the other that limited the overall performance, although the source of these effects was never satisfactorally identified.

\subsubsection{Timeline}

The Achromatic Nulling Testbed began operations in 2003. By 2005 it had explored both the through-focus approach and the use of pairs of dispersive glass plates. From 2005 through 2007 the effort focused on developing and improving the results from the periscope mirror design. However, in May 2007 the Adaptive Nuller demonstrated deeper and more broadband nulls than could be achieved with the ANT (yielding rejection ratios of $82,000: 1$ over a $32 \%$ bandwidth). It seemed clear that the best approach to achromatic phase shifting was through adaptive nulling, and so the operation of the ANT was brought to a close in December 2007. The testbed was dismantled in January 2008.

\subsubsection{Future Technology Development}

Of the methods of achromatic phase shifting that were tested by the ANT, the approach using the periscope mirrors produced the best results. Although these results fell short of the goal of 100,000:1 at 25\% bandwidth, this goal appears to be well within reach of the Adaptive Nuller. An adaptive nuller used in conjunction with a 


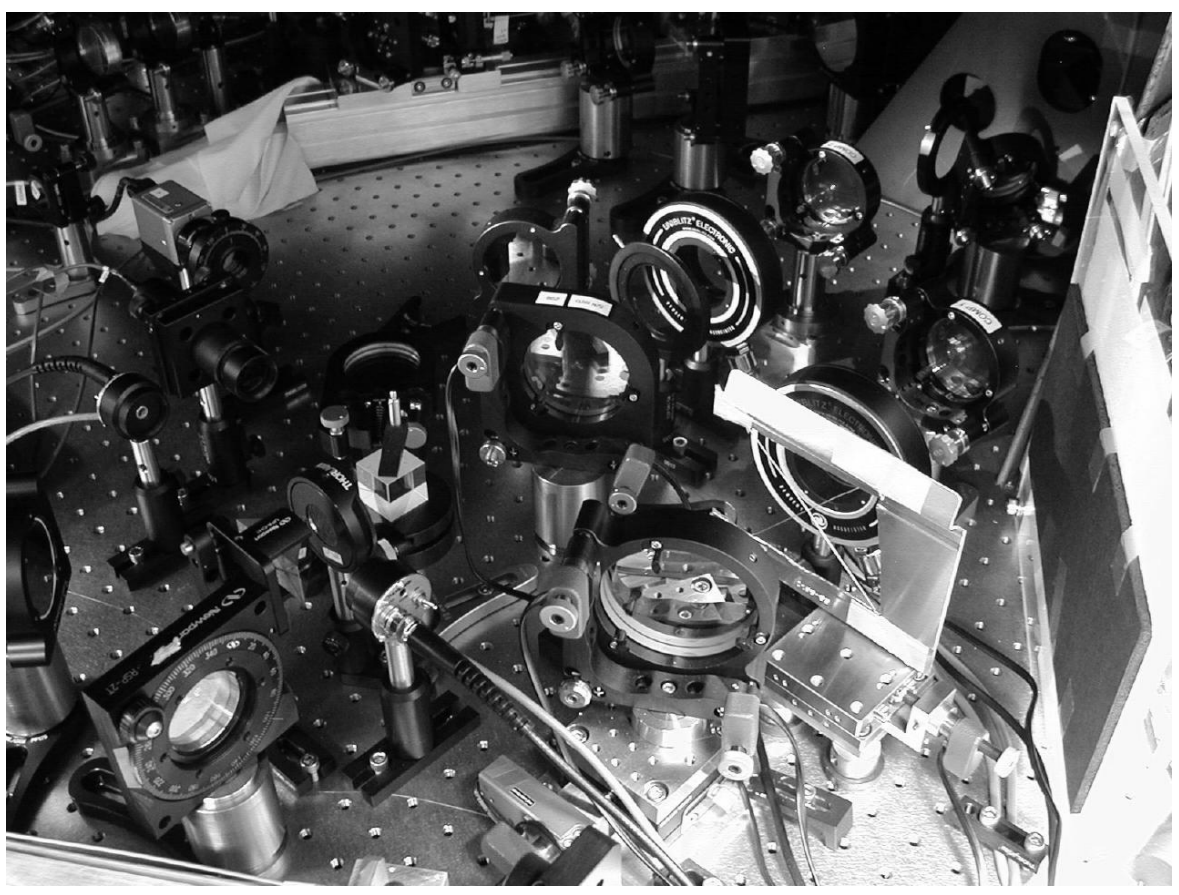

Figure 3. Close up of the beam-combiner of the Achromatic Nulling Testbed, configured for the periscope nuller. The periscope assembly can be seen to the top right. This view includes the compensating plates and the cross-wires for intensity balancing.

periscope phase shifter would appear to be a viable approach, although it is entirely possible that an adaptive nuller alone may be sufficient. Future developments in broadband mid-infrared nulling are now being devoted to improving the performance of the adaptive nulling.

\subsection{Adaptive Nulling}

The Adaptive Nuller was designed to correct phase and intensity variations as a function of wavelength, in each of two linear polarizations. This should allow high performance nulling interferometry, while at the same time substantially relaxing the requirements on the nulling interferometer's optical components. The goal of the testbed was to demonstrate, in a $3 \mu \mathrm{m}$ band centered at a wavelength of $10 \mu \mathrm{m}$, the correction of the intensity difference to less than $0.2 \% \mathrm{rms}(1 \sigma)$ between the interferometer's arms, and at the same time correct the phase difference across the band to $<5 \mathrm{~nm} \mathrm{rms}(1 \sigma)$. This overall correction is consistent with a null depth of $10^{-5}$ (1 part in 100,000) if all other sources of null degradation can be neglected.

\subsubsection{Testbed Description}

The adaptive nuller uses a deformable mirror to adjust amplitude and phase independently in each of about 12 spectral channels. The incident beam is first split into its two linear polarization components, and is dispersed into roughly a dozen spectral channels. The dispersed spectra are then imaged onto a line of pixels on a deformable mirror, so that the piston of each pixel independently adjusts the phase of each channel. Tilt in the orthogonal direction is independently adjusted, which shears the output pupil at that wavelength; this shear, in combination with an output stop, selectively reduces the intensity in that channel. The various component beams are recombined to yield an output beam that has been carefully tuned for intensity and phase in each polarization as a function of wavelength. Optical components then need only be of sufficient quality that the two arms of the interferometer are matched in intensity and phase to within the capture range of the Adaptive Nuller. A photograph of the testbed is shown in Fig. 4. 


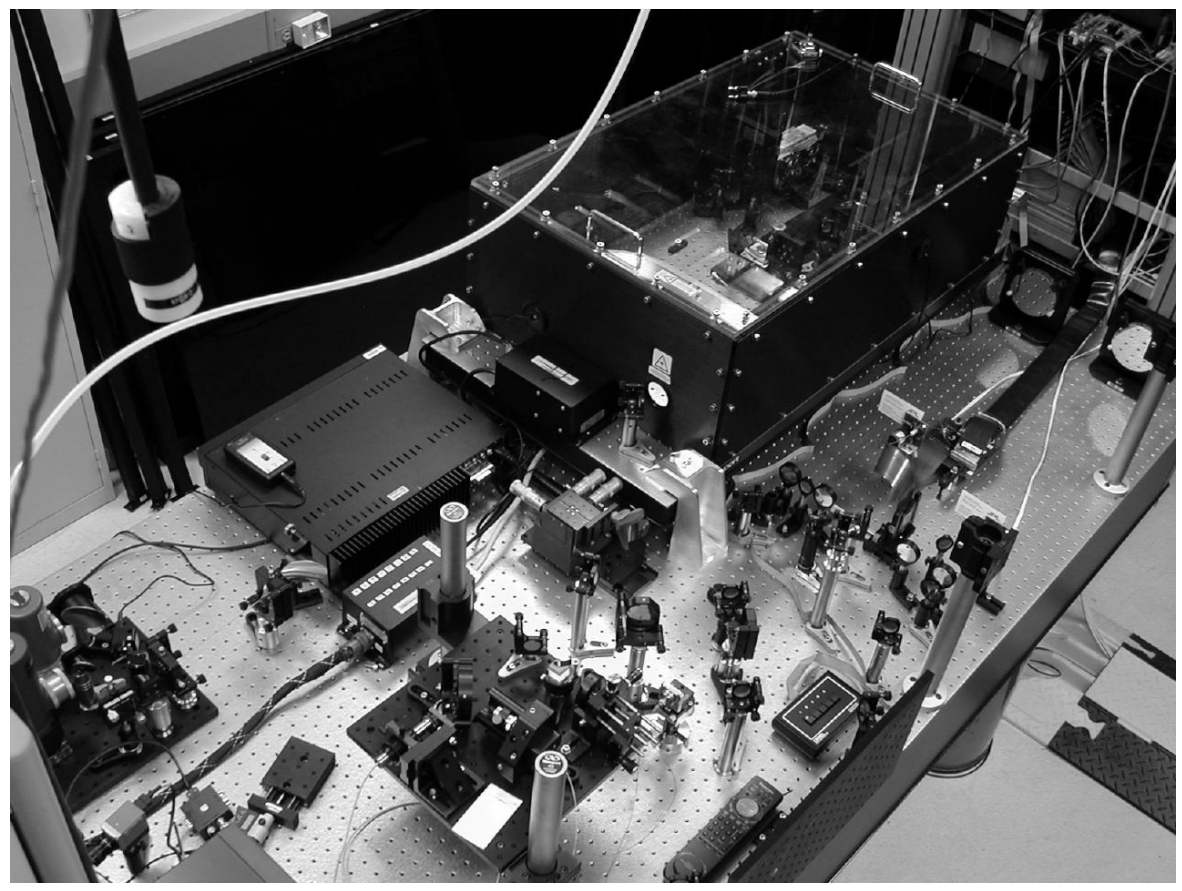

Figure 4. The Adaptive Nuller Testbed (AdN). The Adaptive Nuller demonstrated phase and intensity compensation of beams within a nulling interferometer to a level of $0.12 \% \mathrm{rms}$ in intensity and $<5 \mathrm{~nm} \mathrm{rms}$ in phase. This version of the AdN operates over a wavelength range of 8-12 $\mu \mathrm{m}$. The long-focus parabolas, used in AdN are seen on the upper right.

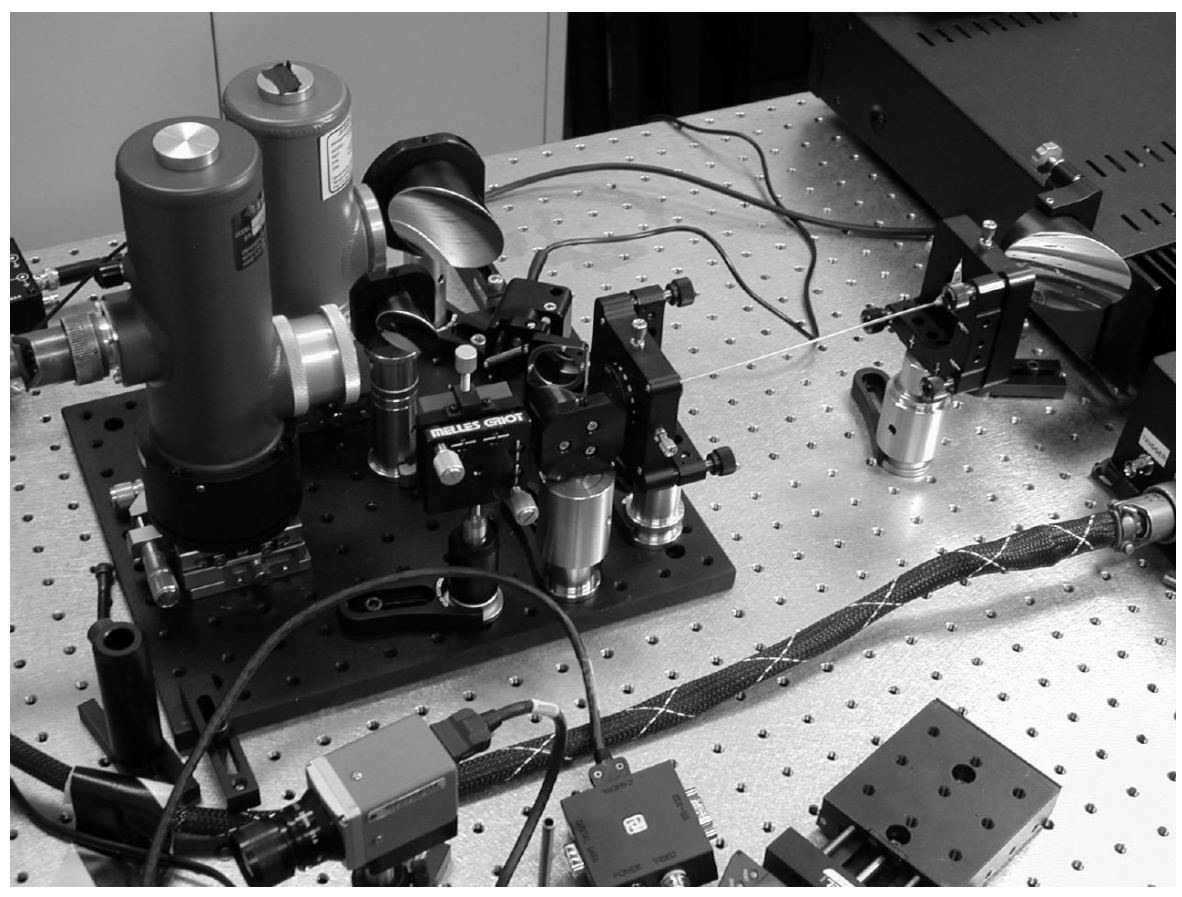

Figure 5. View of the back-end optics of the Adaptive Nuller testbed, showing a Chalcogenide fiber, manufactured by the US Naval Research Laboratory. The fiber is seen as a thin white line, fed by an off-axis parabola, visible toward the upper right. 
In its original concept, the Adaptive Nuller separates the light in each beam into two linear polarizations using Wollaston prisms. Although Wollaston prisms are included in the design, there are no Wollaston prisms in this mid-infrared testbed. The approach to these demonstrations has been that if light in one polarization can be compensated, then it would be straightforward to compensate both using the same overall approach. It is simply a matter of cost. At mid-infrared wavelengths, the only material that can be used to make a Wollaston prism is cadmium selenide (CdSe), which is an extremely expensive material to grow. Prior adaptive nulling experiments at visible wavelengths demonstrated the technique of separating the spectra, and so it wasn't deemed necessary to repeat these experiments at mid-infrared wavelengths. ${ }^{35}$ Surprisingly, the mid-infrared tests with the adaptive nuller to date have been successful without using polarizing filters in the testbed.

\subsubsection{Progress to Date}

The results of this research up until 2007 have been published by Peters et al. ${ }^{4}$ In March/April 2007, the testbed achieved its primary goal of demonstrating phase compensation to better than $5 \mathrm{~nm}$ rms across the $8-12 \mu \mathrm{m}$ band and intensity compensation to better than $0.2 \%$. In June 2008 the mid-infrared Adaptive Nuller demonstrated a null depth of $1.1 \times 10^{-5}$ (93,000:1 rejection ratio), over a bandwidth of $32 \%$. This is the deepest broadband null ever achieved by a mid-infrared nulling interferometer, and almost attains the TPF-I flight requirement. Long term plans now include improving the attainable null depth to better than $1.0 \times 10^{-5}$, and the possible design of a cryogenic version of this testbed. The milestone documents for these tests are as follows:

- "TPF-I Milestone \#1 Whitepaper: Amplitude and Phase Control Demonstration," Edited by R.D. Peters, P.R. Lawson, and O.P. Lay (Jet Propulsion Laboratory, December 2006). http://planetquest.jpl.nasa.gov/TPF-I/TPFI_M1_WhitePaper.pdf

- "TPF-I Milestone \#1 Report: Amplitude and Phase Control Demonstration," Edited by R.D. Peters, P.R. Lawson, and O.P. Lay, JPL Document 38839 (Jet Propulsion Laboratory, July 2007). http://planetquest.jpl.nasa.gov/TPF-I/TPF-I_M1Report_Final_signatures.pdf

- "TPF-I Milestone \#3 Whitepaper: Broadband Startlight Suppression Demonstration," Edited by P.R. Lawson, R.O. Gappinger, R.D. Peters, and O.P. Lay (Jet Propulsion Laboratory, October 2007). http://planetquest.jpl.nasa.gov/TPF-I/TPF-I_M3_WhitepaperFinalSignatures.pdf

\subsection{Planet Detection Testbed}

The Planet Detection Testbed, pictured in Fig. 6, was developed to demonstrate the feasibility of four-beam nulling, the achievement of the required null stability, and the consequent detection of faint planets using approaches similar to the ones contemplated for a flight-mission. The most promising architectures for a flight mission employing synthesis imaging techniques (the X-Array and the Linear Dual-Chopped Bracewell) are fourbeam nulling interferometers that use interferometric chopping to detect planets in the presence of a strong mid-infrared background.

The flight mission would use a phase chopping technique to modulate a sensitivity/fringe pattern around the star. This modulation technique is in many ways similar to the use of a chopper wheel that allows the detection of infrared sources against a thermal background and/or drifting detector offsets. In this case the thermal background on the sky includes the local and exozodiacal light. To achieve this modulation the interferometer uses two nullers each phased to null out the starlight, and a second beam combiner, known as the cross-combiner, which takes the output from the nullers and phases it to form the moving sensitivity pattern. A dark null fringe is fixed over the star and the constructive bright fringes move alternately to each side of the star, thus moving on and off the planet. If there are other planets in the field of view, their signals will also contribute depending on their locations and by rotating the fringe system around the star the whole planetary system can be observed. Signal processing is then used to determine the location of the planets orbiting the star.

The primary objective of the Planet Detection Testbed is to simulate this observing scenario and demonstrate the instrument stability needed to make this process work. Stability is an important requirement of the detection process. The detected signal is the difference in the measured photon flux between the two chop states and this signal has both stochastic and systematic noise components. Integration over time reduces the stochastic 


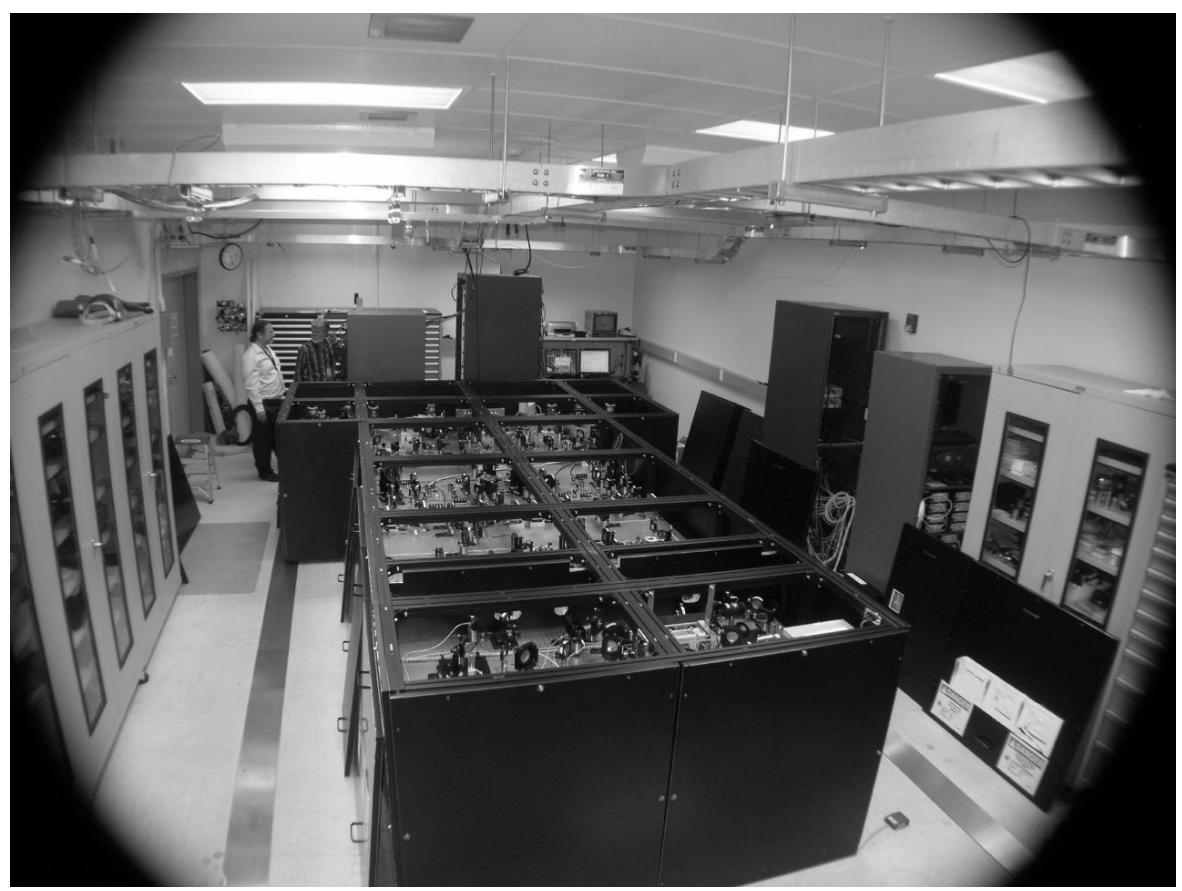

Figure 6. The Planet Detection Testbed (PDT). The PDT is a four-input nulling interferometer that uses $10 \mu \mathrm{m}$ laser light and servo loops to perform experiments related to instability noise, interferometric chopping, and planet detection. The testbed configuration shown here has obtained laser nulls between 500,000:1 and 800,000:1 and detected a simulated planet with a contrast ratio of 2,000,000:1.

components and good instrument stability is needed to minimize systematic components which may appear as low frequency fluctuations with timescales similar to a planet signal. Some of these systematic components can be removed by signal processing using expected correlations across the broadband light spectrum.

\subsubsection{Testbed Description}

The PDT has the following main components: a star and planet source to generate a planet to be observed, a pair of nullers to null out the starlight, and a cross-combiner to allow modulation of the detected planet signal. To provide the necessary stability, the testbed has pointing and shear control systems, laser metrology systems and fringe trackers to maintain the phase on the star.

The PDT combines simulated star and planet beams in pairs to produce four star/planet beams that enter the testbed as if detected through four separate telescopes. Delay lines are used for the planet light relative to the star-light to simulate the path delays that would be observed as the array rotates with respect to the star/planet system. Two beam pairs are nulled and then cross-combined. A $\pi$ phase shift is introduced into one of each beam pair by a combination of optical path differences in glass and air. The beam-pairs are chopped using a standard infrared approach and the difference in chop-states is recorded. The testbed is described in detail in the milestone whitepaper by Martin et al. (below), and in a companion paper presented at this conference. ${ }^{30}$

\subsubsection{Progress to Date}

Following successful tests in 2005, the PDT was rebuilt to include tilt and shear sensors that in February 2007 demonstrated intensity stability in each arm of the interferometer to better than $0.2 \%$. The PDT is now fully operational. Experiments in 2008 have yielded null pairs with null depths between 500,000:1 and 800,000:1. In April 2008 the milestone criteria for the initial planet detection demonstrations were established:

- "Exoplanet Interferometry Milestone \#4 Whitepaper: Planet Detection Demonstration," Edited by S. R. Martin, A. J. Booth, O. P. Lay, and P. R. Lawson (Jet Propulsion Laboratory, May 2008). 
1. Detect a planet at a contrast of $\leq 10^{-6}$ relative to the star at a signal to noise ratio of $\geq 10$.

2. Show residual starlight suppression from phase chopping, averaging and rotation $\geq 100$.

3. The tests in (1) and (2) must each run for a total duration of 10,000 s and may include one or more planet rotations at timescales $\geq 2,000 \mathrm{~s}$.

4. The tests in (1) and (2) must be satisfied simultaneously on three separate occasions with at least 48 hours between each demonstration.

This constitutes a demonstration of three of the four main parts of the starlight suppression technique: deep interferometric nulling, phase chopping and formation rotation to modulate the planet. The fourth part is the extraction of a planet signal using a broadband spectral filtering technique. ${ }^{29}$ The current plan is to attempt this additional milestone with the PDT in 2009.

\section{TECHNOLOGY FOR FORMATION FLYING}

\subsection{Formation Control Testbed}

The Formation Control Testbed (FCT) was built to provide an end-to-end autonomous formation flying system in a ground-based laboratory. The FCT provides an environment for system-level demonstration and validation of formation control algorithms. The algorithms are validated using multiple floating test robots that emulate real spacecraft dynamics. The goal is to demonstrate algorithms for formation acquisition, formation maneuvering, fault-tolerant operations, as well as collision-avoidance maneuvering.

\subsubsection{Testbed Description}

The FCT is comprised of two robots with flight-like hardware and dynamics, a precision flat floor for the robots to operate on, ceiling-mounted artificial stars for robot attitude sensing and navigation, and a "ground control" room for commanding the robots and receiving telemetry. The robots and part of the flat floor are shown in Fig. 7. The layout of the FCT emulates the environment of a formation of telescopes that is restricted to maneuvering in the same plane in space, normal to the direction of the target star.

To be as flight-like as possible, each robot is equipped with a typical single-spacecraft attitude control suite of reaction wheels, gyros, and a star tracker. Thrusters are also available for attitude control. Each robot has a lower translational platform and an upper attitude platform. The attitude platform is the "spacecraft" and is completely disconnected from the translational platform.

* The attitude platform/spacecraft houses the avionics, actuators, sensors, inter-robot and "ground"-to-robot wireless communication antennae, and the spacecraft processors.

* The translational platform provides both translational and rotational degrees of freedom to the attitude platform via (i) linear air bearings (the black, circular pads at the base of each robot) that allow the entire robot to float freely on the flat floor, and (ii) a spherical air bearing at the top of the vertical stage (the black, vertical cylinders).

Each robot therefore has five degrees-of-freedom: two in translation and three in rotation. The robots can translate wherever necessary on the flat floor; however, the pitch and roll axes of each robot's motion are limited to \pm 30 degrees (a physical limitation of the spherical air bearings). The formation algorithms are designed for all six degrees-of-freedom, and for the vertical translational degree of freedom, telescoping vertical stages with $0.5 \mathrm{~m}$ of travel are being retrofitted in 2008. The first of these is shown in Fig. 7. The vertical air bearing have a range of $\pm 25 \mathrm{~cm}$.

The FCT is housed in the former Celestarium, which had been used to calibrate star trackers. The FCT has a $40 \mathrm{ft}$. diameter circular floor space and a $25 \mathrm{ft}$. high, dome-like ceiling. This ceiling is ideal for mounting artificial reference stars. The precision flat floor that the robots operate on is contained within the $40 \mathrm{ft}$. diameter floor space. The flat floor consists of fourteen $4 \mathrm{ft} . \times 12 \mathrm{ft}$. metal panels ground to a flatness of $0.002 \mathrm{in}$. Each panel is mounted on a support structure that has coarse and vernier leveling screws. Periodic laser surveys of the floor are used to ensure that the steps between the panels are less than 0.001 inch and the floor slope is less than 
120 microradians. The resulting $7.3 \mathrm{~m} \times 8.5 \mathrm{~m}$ flat floor is sufficient to demonstrate nominal TPF-I formation maneuvers, such as formation rotations and collision avoidance.

To provide artificial stars, down-looking infrared LEDs are mounted on the ceiling of the Celestarium. The star tracker on each robot measures the direction (two angles) to at least three stars. From this information, the attitude and position of a robot can be determined with respect to the inertial room frame. The position of a robot can also be determined because the stars are in the near-field, and hence, the direction to an LED changes as a robot moves.

\subsubsection{Results}

In 2007 the Formation Control Testbed demonstrated its milestone for precision maneuvers using two robots, showing autonomous initialization, maneuvering, and operation in a collision free manner. ${ }^{5,6}$ The key maneuver that was demonstrated was representative of TPF-I science observations. Repeated experiments with the robots demonstrated formation rotations through greater than 90 degrees at ten times the flight rotation rate while maintaining a relative position control to $5 \mathrm{~cm}$ rms. Although the achievable resolution in these experiments was limited by the noise environment of the laboratory, it was nonetheless demonstrated (through modeling) that in the relatively noise-free environment of space, the performance of these algorithms would exceed TPF-I flight requirements.

\subsubsection{Timeline}

Planning for the Formation Control Testbed began in 2003. The first robot for the FCT was commissioned in September 2004 and the second in January 2006. In May 2006 the integration of the FACS software was

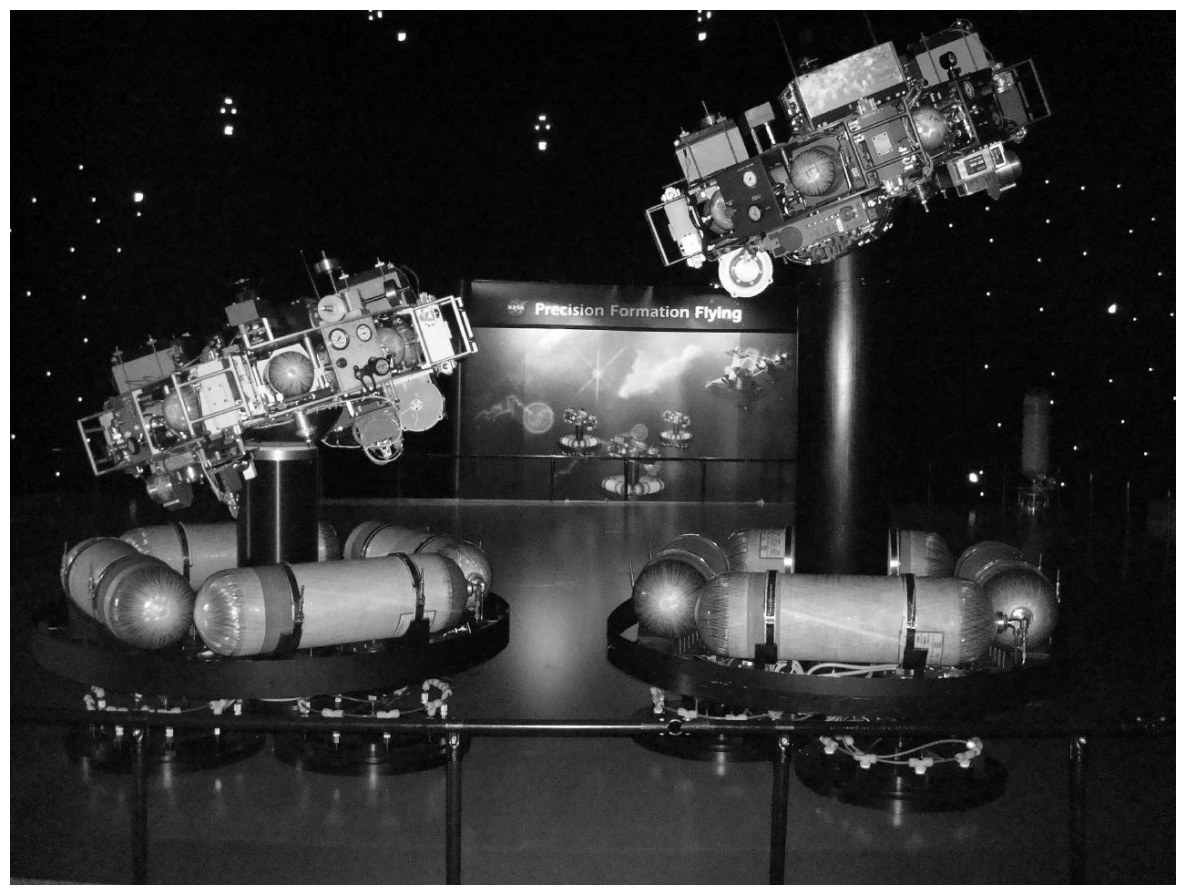

Figure 7. The Formation Control Testbed (FCT). Shown here are the two robots of the FCT. Each robot carries cannisters of compressed air that allow them to float off a polished metal floor. The floor is flat to within 2 one-thousandths of an inch and spans a much larger area than shown here. The robots carry a platform (shown tilted for each robot) that is supported on a spherical ball bearing, also driven with compressed air so that the support of the platform is entirely frictionless. The first vertical stage is shown installed in the robot on the right hand side of the photograph. The second vertical stage will be installed in the other robot in June 2008. The robots serve as the hardware interface and testing ground for flight software developed for space applications in formation flying. 
successfully completed for the robots, enabling them to operate in a formation mode for the first time. In late 2006 and early 2007 the robots underwent extensive hardware upgrades in preparation for their performance milestone. The experiments for that milestone were conducted in September 2007. In November 2007 the first vertical stage was commissioned for one of the robots. The vertical stage for the second robot is anticipated in June of 2008. The research sponsored by NASA for TPF was concluded in January 2008. The Formation Control Testbed continues to be developed in support of DARPA and its F-6 Program. Details of the performance milestone are contained in the following documents:

- "TPF-I Milestone \#2 Whitepaper: Formation Control Performance Demonstration," Edited by D.P. Scharf (Jet Propulsion Laboratory, May 2007). http://planetquest.jpl.nasa.gov/TPF-I/TPFI_M2_WhitePaper_Final.pdf

- "TPF-I Technology Milestone \#2 Report: Formation Control Performance Demonstration," Edited by D.P. Scharf and P. R. Lawson, JPL Pub. 08-11 (Jet Propulsion Laboratory, January 2008). http://planetquest.jpl.nasa.gov/TPF-I/TPFI_M2_ReportV3.pdf

\subsubsection{Future Technology Development}

The next steps for technology maturation that could be done using the robots within the FCT include demonstrating new capabilities such as (1) reactive collision avoidance, (2) formation fault detection, and (3) autonomous reconfiguration and retargeting maneuvers. Using the real-time simulation environment of FAST we could also need to demonstrate the performance with full formation-flight complexity, that is with five interacting spacecraft showing synchronized rotations, autonomous reconfigurations, fault detection, and collision avoidance.

Although NASA has no immediate plans to continue supporting formation flying research, national agencies in Europe are actively advancing the technology, with flight missions starting in 2009-2014. The European Space Agency and national space agencies in Europe have a program of precursor missions to gain experience in formation flying to support XEUS and Darwin. In 2009 the Swedish Space Agency will launch the Prisma mission. This is primarily a rendezvous and docking mission, but will also test RF metrology designed for Darwin. In 2012 ESA plans to launch Proba-3, which is specifically a technology precursor to XEUS, and will include optical metrology loops for sub-millimeter range control over a 30-m spacecraft separation. The French and Italian space agencies are planning to launch Simbol-X in 2014. Simbol-X is an X-ray science mission with an architecture very similar to XEUS, but with a 20-m spacecraft separation. Simbol-X should enter Phase B of development in the summer of 2008.

The greatest advance in maturing technology for formation flying would certainly be to have a modest-scale technology mission devoted to verifying and validating guidance and control algorithms, and to further include the interferometric combination of starlight from separated platforms. A ground-based facility such as the FCT will continue to provide the means to test and improve real-time formation-flying algorithms as the technology matures, even while the technology is being proven in space.

\section{SUMMARY}

Technology development for the Terrestrial Planet Finder Interferometer is proceeding well. The technology for nulling interferometry is reaching maturity, as laboratory results have approached the levels required for a flight mission. The Adaptive Nuller has also appeared as the clear choice of techniques for achromatic phase shifting. Tests in 2007 at JPL demonstrated that the algorithms for formation flying are also ready for flight. Future technology demonstrations may include the following:

1. Demonstrate broadband mid-infrared nulling ( $25 \%$ bandwidth or greater) at a mean level of $10^{-5}$ for several hours (Milestone \#3).

2. Demonstrate additional mid-infrared noise suppression using chopping, rotation, and averaging in a fourbeam interferometer (Milestone \#4). 
3. Demonstrate the final level of noise suppression using broadband mid-infrared spectral filtering to reject instability noise (Milestone \#5).

4. Verify and validate the short and long wavelength performance of mid-infrared spatial filters across the 6-20 $\mu \mathrm{m}$ band, possibly including cryogenic testing of fibers.

5. Show improved throughput with spatial filters, possibly using photonic crystal fibers.

6. Demonstrate algorithms for collision avoidance of formations of multiple spacecraft.

7. Demonstrate fault-tolerant algorithms for formation flying.

8. Demonstrate in space the validation of RF and optical metrology between spacecraft.

9. Validate thruster technology that would be suitable for planet finding maneuvers.

10. Demonstrate in space $(300 \mathrm{~K})$ testing of interferometry technology for long-baseline fixed-platform and multi-platform interferometers.

11. Demonstrate and/or inherit cryogenic technology for passive cooling of structures and optics, and active cooling of detectors.

12. Demonstrate the cryogenic performance of selected interferometer mechanisms, including delay lines and tilt servos.

Experiments are now underway to demonstrate planet signal extraction using chopping and array rotation. Further experiments are anticipated in 2009 to also demonstrate spectral filtering techniques. In the near future, experience with formation flying in space and practical experience with the cryogenic systems of JWST will put TPF-I on a solid foundation by the first half of the coming decade.

\section{ACKNOWLEDGMENTS}

This work was conducted at the Jet Propulsion Laboratory, California Institute of Technology, under contract with the National Aeronautics and Space Administration. Reference in this paper to any specific commercial product, process, or service by trade name, trademark, manufacturer, or otherwise, does not constitute or imply its endorsement by the United States Government or the Jet Propulsion Laboratory, California Institute of Technology.

\section{REFERENCES}

1. P. R. Lawson, A. Ahmed, R. O. Gappinger, A. Ksendzov, O. P. Lay, S. R. Martin, R. D. Peters, D. P. Scharf, J. K. Wallace, and B. Ware, "Terrestrial Planet Finder Interferometer: Technology status and plans," in Advances in Stellar Interferometry, J. Monnier, M. Schöller, and W. Danchi, eds., Proc. SPIE 6268, p. $626828,2006$.

2. P. R. Lawson, O. P. Lay, S. R. Martin, C. A. Beichman, K. J. Johnston, W. C. Danchi, R. O. Gappinger, S. L. Hunyadi, A. Ksendzov, B. Mennesson, R. D. Peters, D. P. Scharf, E. Serabyn, and S. C. Unwin, "Terrestrial Planet Finder Interferometer: 2006-2007 progress and plans," in Techniques and Instrumentation for Detection of Exoplanets III, D. R. Coulter, ed., Proc. SPIE 6693, p. 669308, 2007.

3. R. D. Peters, O. P. Lay, and M. Jeganathan, "Adaptive nulling in the mid-IR for the Terrestrial Planet Finder Interferometer," in Techniques and Instrumentation for Detection of Exoplanets III, D. R. Coulter, ed., Proc. SPIE 6693, p. 669315, 2007.

4. R. D. Peters, O. P. Lay, and M. Jeganathan, "Broadband phase and intensity compensation with a deformable mirror for an interferometric nuller," Appl. Opt. 47, p. in press, 2008.

5. D. P. Scharf, J. A. Keim, F. Y. Hadaegh, E. G. Benowitz, and P. R. Lawson, "Flight-like ground demonstration of precision formation flying spacecraft," in Techniques and Instrumentation for Detection of Exoplanets III, D. R. Coulter, ed., Proc. SPIE 6693, p. 669307, 2007. 
6. D. P. Scharf, F. Y. Hadaegh, J. A. Keim, A. C. Morfopoulos, A. Ahmed, Y. Brenman, A. Vafaei, J. F. Shields, C. F. Bergh, and P. R. Lawson, "Flight-like ground demonstrations of precision maneuvers for spacecraft formations," AIAA Journal of Guidance, Control, and Dynamics 31, p. submitted, 2008.

7. S. R. Martin, "TPF-Emma: concept study of a planet finding space interferometer," in Techniques and Instrumentation for Detection of Exoplanets III, D. R. Coulter, ed., Proc. SPIE 6693, p. 669309, 2007.

8. C. S. Cockell, A. Leger, M. Fridlund, T. Herbst, L. Kaltenegger, O. Absil, C. Beichman, W. Benz, M. Blanc, A. Brack, A. Chelli, L. Colangeli, H. Cottin, V. Coude du Foresto, W. Danchi, D. Defrere, J.-W. den Herder, C. Eiroa, J. Greaves, T. Henning, K. Johnston, H. Jones, L. Labadie, H. Lammer, R. Launhardt, P. Lawson, O. P. Lay, J.-M. LeDuigou, R. Liseau, F. Malbet, S. R. Martin, D. Mawet, D. Mourard, C. Moutou, L. Mugnier, F. Paresce, A. Quirrenbach, Y. Rabbia, J. A. Raven, H. J. A. Rottgering, D. Rouan, N. Santos, F. Selsis, E. Serabyn, H. Shibai, M. Tamura, E. Thiebaut, F. Westall, and J. G. White, "Darwin - a mission to detect, and search for life on, extrasolar planets," Astrobiology 8, p. accepted, 2008.

9. H. Bokhove, J. P. Kappelhof, H. J. P. Vink, L. L. A. Vosteen, and Z. Sodnik, "Broadband nulling using a prism phase shifter," in Towards Other Earths: Darwin/TPF and the Search for Extrasolar Terrestrial Planets, M. Fridlund and T. Henning, eds., SP-539, pp. 367-369, European Space Agency, (Noordwijk, The Netherlands), 2003.

10. F. Brachet, A. Labéque, A. Léger, M. Ollivier, C. L. V. Hervier, B. Chazelas, B. Pellet, T. Lépine, and C. Valette, "Nulling interferometry for the Darwin misison: Polychromatic laboratory test bench," in New Frontiers in Stellar Interferometry, W. A. Traub, ed., Proc. SPIE 5491, pp. 991-998, 2004.

11. C. B. X. Rejeaunier, Y. Rabbia, C. Ruilier, M. Barillot, L. Lierstuen, and J. M. Perdigues Armeng, "Multiaxial nulling interferometry: Demonstration of deep nulling and investigations of polarization effects," in Advances in Stellar Interferometry, J. D. Monnier, M. Schöller, and W. C. Danchi, eds., Proc. SPIE 6268, p. $626819,2006$.

12. C. Buisset, X. Rejeaunier, Y. Rabbia, and M. Barillot, "Stable deep nulling in polychromatic unpolarized light with multi-axial beam combination," Appl. Opt. 46, pp. 7817-7822, 2007.

13. K. Ergenzinger, R. Flatscher, U. Johann, R. Vink, and Z. Sodnik, "EADS Astrium nulling interferometer breadboard for Darwin and GENIE," in Proc. Int. Conf. Space Optics, M. Fridlund and T. Henning, eds., SP-554, pp. 223-230, European Space Agency, (Noordwijk, The Netherlands), 2004.

14. P. Haguenauer and E. Serabyn, "Deep nulling of laser light with a single-mode-fiber beam combiner," Appl. Opt. 45, pp. 2749-2754, 2006.

15. S. R. Martin, R. O. Gappinger, F. M. Loya, B. P. Mennesson, S. L. Crawford, and E. Serabyn, "A midinfrared nuller for the Terrestrial Planet Finder: Design, progress, and results," in Techniques and Instrumentation for Detection of Exoplanets, D. R. Coulter, ed., Proc. SPIE 5170, pp. 144-154, 2003.

16. S. R. Martin, E. Serabyn, and G. J. Hardy, "Deep nulling of laser light in a rotational shearing interferometer," in Interferometry for Optical Astronomy II, W. A. Traub, ed., Proc. SPIE 4838, pp. 656-667, 2003.

17. S. Martin, P. Szwaykowski, and F. Loya, "Testing exo-planet signal extraction using the Terrestrial Planet Finder Planet Detection Testbed," in Techniques and Instrumentation for Detection of Exoplanets II, D. R. Coulter, ed., Proc. SPIE 5905, p. 590508, 2005.

18. S. Martin, "The flight instrument design for the Terrestrial Planet Finder Interferometer," in Techniques and Instrumentation for Detection of Exoplanets II, D. R. Coulter, ed., Proc. SPIE 5905, pp. 21-35, 2005.

19. B. Mennesson, S. L. Crawford, E. Serabyn, S. Martin, M. Creech-Eakman, and G. Hardy, "Laboratory performance of the Keck Interferometer nulling beam combiner," in Towards Other Earths: Darwin/TPF and the Search for Extrasolar Terrestrial Planets, M. Fridlund and T. Henning, eds., SP-539, pp. 525-529, European Space Agency, (Noordwijk, The Netherlands), 2003.

20. B. Mennesson, P. Haguenauer, E. Serabyn, and K. Liewer, "Deep broad-band infrared nulling using a singlemode fiber beam combiner and baseline rotation," in Advances in Stellar Interferometry, J. D. Monnier, M. Schöller, and W. C. Danchi, eds., Proc. SPIE 6268, p. 626830, 2006.

21. R. M. Morgan, J. H. Burge, and N. J. Woolf, "Final laboratory results of visible nulling with dielectric plates," in Interferometry for Optical Astronomy II, W. A. Traub, ed., Proc. SPIE 4838, pp. 644-655, 2003. 
22. M. Ollivier, Contribution à la Recherche d'Exoplanétes: Coronagraphie Interfèrentielle pour la Mission Darwin. PhD thesis, University Paris XI, Orsay, France, 1999.

23. R. Samuele, J. K. Wallace, E. Schmidtlin, M. Shao, B. M. Levine, and S. Fregoso, "Experimental progress and results of a visible nulling coronagraph," in 2007 IEEE Aerospace Conference, p. paper 1333, 2007.

24. E. Schmidtlin, J. K. Wallace, R. Samuele, B. M. Levine, and M. Shao, "Recent progress of visible light nulling and first 1 million null result," in Direct Imaging of Exoplanets: Science and Techniques, C. Aime and F. Vakili, eds., Proc. IAU Colloq. 200, pp. 353-360, 2006.

25. E. Serabyn, J. K. Wallace, G. J. Hardy, E. G. H. Schmidtlin, and H. T. Nguyen, "Deep nulling of visible laserlight," Appl. Opt. 38, pp. 7128-7132, 1999.

26. L. L. A. Vosteen, H. J. P. Vink, H. van Brug, and H. Bokhove, "Achromatic phase-shifter breadboard extensions," in Techniques and Instrumentation for Detection of Exoplanets II, D. R. Coulter, ed., Proc. SPIE 5905, p. 59050A, 2005.

27. J. K. Wallace, G. Hardy, and E. Serabyn, "Deep and stable interferometric nulling of broadband light with implications for observing planets around nearby stars," Nature 406, pp. 700-702, 2000.

28. V. Weber, M. Barillot, P. Haguenauer, P. Kern, I. Schanen-Duport, P. Labeye, L. Pujol, and Z. Sodnik, "Nulling interferometer based on an integrated optics combiner," in New Frontiers in Stellar Interferometry, W. A. Traub, ed., Proc. SPIE 5491, pp. 842-850, 2004.

29. O. P. Lay, "Removing instability noise in nulling interferometers," in Advances in Stellar Interferometry, J. D. Monnier, M. Schöller, and W. C. Danchi, eds., Proc. SPIE 6268, p. 62681A, 2006.

30. A. J. Booth, S. R. Martin, and F. M. Loya, "Exoplanet Exploration Program, Planet Detection Testbed: Latest results of planet light detection in the presence of starlight," in Optical and Infrared Interferometry, M. Schöller, W. C. Danchi, and F. Delplancke, eds., Proc. SPIE 7013, p. these proceedings, 2008.

31. A. Ksendzov, O. Lay, S. Martin, J. S. Sanghera, L. E. Busse, W. H. Kim, P. C. Pureza, and V. Q. Nguyen, "Characterization of mid-infrared single mode fibers as modal filters," Appl. Opt. 46, pp. 7957-7962, 2007.

32. A. Ksendzov, T. Lewi, O. Lay, S. Martin, R. Gappinger, P. Lawson, R. Peters, S. Shalem, A. Tsun, and A. Katzir, "Modal filtering in mid-infrared using single mode silver halide fibers," Appl. Opt. 47, p. submitted, 2008.

33. T. Lewi, A. Ksendzov, S. R. Martin, O. P. Lay, S. Shalem, A. Tsun, A. Katzir, and P. R. Lawson, "Silver halide single mode fibers for modal filtering in the middle infrared," in Optical and Infrared Interferometry, M. Schöller, W. C. Danchi, and F. Delplancke, eds., Proc. SPIE 7013, p. these proceedings, 2008.

34. R. O. Gappinger, R. T. Diaz, P. R. Lawson, S. R. Martin, O. P. Lay, J. K. Wallace, and F. M. Loya, "Experimental evaluation of achromatic phase shifters for mid-infrared starlight suppression," Appl. Opt. 47, p. submitted, 2008.

35. R. D. Peters, A. Hirai, M. Jeganathan, and O. P. Lay, "Near-IR demonstration of adaptive nuller based on deformable mirror," in New Frontiers in Stellar Interferometry, W. A. Traub, ed., Proc. SPIE 5491, pp. 1630-1638, 2004. 